\title{
Impact of Electrostatic Coupling on Monolithic 3D-enabled Network on Chip
}

\author{
DONGJIN LEE, SOURAV DAS, JANARDHAN RAO DOPPA, and \\ PARTHA PRATIM PANDE, Washington State University \\ KRISHNENDU CHAKRABARTY, Duke University
}

\begin{abstract}
Monolithic-3D-integration (M3D) improves the performance and energy efficiency of 3D ICs over conventional through-silicon-vias-based counterparts. The smaller dimensions of monolithic inter-tier vias offer high-density integration, the flexibility of partitioning logic blocks across multiple tiers, and significantly reduced total wire-length enable high-performance and energy-efficiency. However, the performance of M3D ICs degrades due to the presence of electrostatic coupling when the inter-layer-dielectric thickness between two adjacent tiers is less than $50 \mathrm{~nm}$. In this work, we evaluate the performance of an M3D-enabled Networkon-chip (NoC) architecture in the presence of electrostatic coupling. Electrostatic coupling induces significant delay and energy overheads for the multi-tier NoC routers. This in turn results in considerable performance degradation if the NoC design methodology does not incorporate the effects of electrostatic coupling. We demonstrate that electrostatic coupling degrades the energy-delay-product of an M3D NoC by $18.1 \%$ averaged over eight different applications from SPLASH-2 and PARSEC benchmark suites. As a countermeasure, we advocate the adoption of electrostatic coupling-aware M3D NoC design methodology. Experimental results show that the coupling-aware $\mathrm{M} 3 \mathrm{D} \mathrm{NoC}$ reduces performance penalty by lowering the number of multi-tier routers significantly.
\end{abstract}

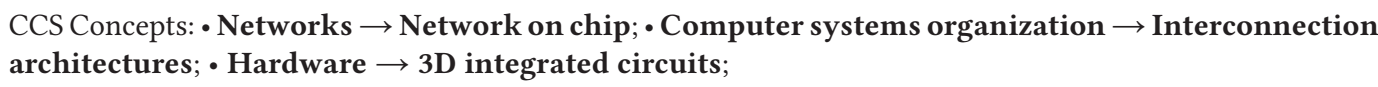

Additional Key Words and Phrases: Monolithic 3D, electrostatic coupling, NoC, latency, energy, EDP, performance, energy efficiency, optimization

\section{ACM Reference format:}

Dongjin Lee, Sourav Das, Janardhan Rao Doppa, Partha Pratim Pande, and Krishnendu Chakrabarty. 2019. Impact of Electrostatic Coupling on Monolithic 3D-enabled Network on Chip. ACM Trans. Des. Autom. Electron. Syst. 24, 6, Article 62 (September 2019), 22 pages.

https://doi.org/10.1145/3357158

\footnotetext{
This work is supported by the US National Science Foundation (NSF) grants CNS-1564014 and CCF 1514269 and USA Army Research Office grant W911NF-17-1-0485.

Author's addresses: D. Lee, S. Das, J. R. Doppa, and P. P. Pande, School of Electrical Engineering and Computer Science, Washington State University, Box 642752, Pullman, Washington 99164; emails: \{dongjin.lee, sourav.das, jana.doppa, pande\}@wsu.edu; K. Chakrabarty, Electrical and Computer Engineering, Duke University, Box 90291, 130 Hudson Hall, Durham, NC 27708; email: krishnendu.chakrabarty@duke.edu.

Permission to make digital or hard copies of all or part of this work for personal or classroom use is granted without fee provided that copies are not made or distributed for profit or commercial advantage and that copies bear this notice and the full citation on the first page. Copyrights for components of this work owned by others than ACM must be honored. Abstracting with credit is permitted. To copy otherwise, or republish, to post on servers or to redistribute to lists, requires prior specific permission and/or a fee. Request permissions from permissions@acm.org. (C) 2019 Association for Computing Machinery.

1084-4309/2019/09-ART62 \$15.00

https://doi.org/10.1145/3357158
} 


\section{INTRODUCTION}

Three-dimensional Network-on-Chip (3D NoC) has emerged as a promising approach to design high-performance and energy-efficient manycore architectures. It combines the benefits of high integration density and energy efficiency of 3D integration along with the scalability of NoC to offer unprecedented performance gain (Khayambashi et al. 2015; Das et al. 2015). There are principally two different 3D integration technologies, viz. through-silicon-via (TSV) and monolithic 3D (M3D). In TSV-based 3D integration, each planar layer is fabricated separately and stacked on top of each other, and adjacent layers are connected through TSV-based vertical links (Davis et al. 2005; Liu et al. 2011). The dimensions of TSVs are significantly larger than the regular Cu-vias. Hence, the corresponding parasitic values of the TSVs are higher too. Consequently, TSV-based links dissipate more energy than traditional planar links (Van der Plas et al. 2011). In addition, fabrication-induced defects, low yield rates, bonding faults, and failure of TSVs over time due to workload-induced stress are of further concern (Knickerbocker et al. 2008; Marinissen and Zorian 2009).

However, for M3D-based integration, vertical interconnects are designed with monolithic-intertier vias (MIVs), which are similar to regular $\mathrm{Cu}$-vias and offer $1,000 \times$ reduction in physical dimensions compared to TSV (Lee et al. 2012). Additionally, due to the presence of thin inter-layer dielectric (ILD) in between planar tiers (the layer thickness ranges from 20 to 300nm for M3D), M3D offers significantly higher integration density than the TSV-based counterpart (Lee et al. 2012; Batude et al. 2011; Yu et al. 2016). By exploiting the flexibility of transistor- and gate-level partitioning, logic blocks can be extended over multiple tiers in M3D-based designs. From the NoC design perspective, it opens up the possibility of designing cores and associated routers extended over multiple tiers by utilizing MIVs. Compared to TSV-based 3D-ICs, M3D enabled ICs achieve significant reduction in effective parasitic capacitance, and thereby gives rise to improved performance and energy-efficiency (Batude et al. 2011; Acharya et al. 2016).

Prior work on M3D mostly focused on the performance and power benefits of sequential integration of transistor tiers and nanoscale vertical interconnects (Lee et al. 2012; Batude et al. 2011; Acharya et al. 2016). The minuscule size monolithic vias (MIVs) and reduced wirelength of M3D-integration results in around 30\% performance improvement for small logic blocks over conventional 2D counterparts (Lee et al. 2013). However, as the ILD thickness between planar tiers in M3D gets thinner with advancing technology, the electrostatic coupling between stacked devices in the fully depleted FDSOI technology affects the timing characteristics of logic blocks from adjacent tiers. The resultant electrostatic coupling between the transistors from adjacent tiers were carefully engineered to design 4T-M3D RAM cells that showed significant resiliency and reduction of area overhead over conventional planar 6T-RAM cells (Batude et al. 2008; Andrieu et al. 2017). However, for the logic circuits, the resulting variation in delay causes timing violations in critical paths and consequently, performance of M3D-based circuits degrades (Yu et al. 2016; Batude et al. 2008; Koneru et al. 2017). In an M3D NoC, this phenomenon negatively affects the critical stage of the router block and introduces additional timing penalty. This results in increased latency and higher overall energy consumption. Hence, it is necessary to consider the effects of the electrostatic coupling while designing the M3D NoC. In this work, our aim is to introduce a system-level design methodology that can circumvent the negative impacts of electrostatic coupling at the transistor/circuit level.

In a 3D NoC, suitable placement of the routers and the associated links governs the overall achievable performance (e.g., latency and energy consumption). In this article, we advocate that the design and optimization of M3D-enabled NoC should incorporate the effects of electrostatic coupling to exploit the true benefits of M3D integration. More specifically, the energy efficiency offered by the MIVs in M3D integration and the high performance ensured by the presence of 


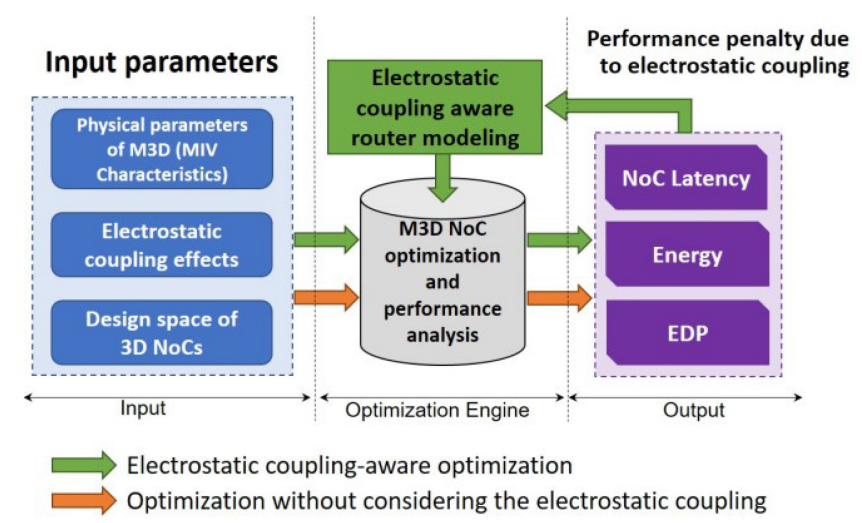

Fig. 1. Illustration of the electrostatic coupling-aware design and evaluation methodology for M3D NoC. The orange color arrow is used to denote the performance degradation analysis of 3D NoC without considering the "electrostatic coupling aware optimization process," while the green arrow refers to performance analysis incorporating "electrostatic coupling aware optimization process."

multi-tier routers need to be considered in conjunction with the timing penalty that electrostatic coupling may introduce. The design of a high performance and energy-efficient M3D NoC is a multi-objective (i.e., network latency, energy and EDP) optimization problem. Therefore, designing an efficient M3D NoC should simultaneously optimize for the multiple objectives while considering the benefits of M3D-integration. To solve multi-objective optimization problems (MOO), we employ Archived Multi-Objective Simulated Annealing (AMOSA) (Bandyopadhyay et al. 2008) to explore the design space to optimize the placement of both links and routers. During optimization, we consider the benefits of M3D-integration and the effects of electrostatic coupling to ensure low latency and low energy consumption for the M3D-enabled NoC.

To this end, our contributions are threefold:

- We study the effects of electrostatic coupling on the performance of M3D NoC depending on the ILD thickness. We demonstrate that the performance of M3D-NoC degrades on an average by $18 \%$ for designs that are unaware of the effects of electrostatic coupling.

- We employ simulated annealing-based multi-objective optimization algorithm and incorporate the effects electrostatic coupling during the NoC design. This in turn minimizes the performance penalty of M3D NoC caused by electrostatic coupling.

- We demonstrate that by adopting the electrostatic coupling-aware design, we need to significantly reduce the allowable number of multi-tier routers. This in turn reduces the performance penalty arising due to coupling.

Figure 1 shows the overall workflow diagram of this approach. The NoC design and optimization depend on the M3D physical parameters. We consider three sets of input parameters, e.g., (i) physical characteristics of M3D integration including the MIV characteristics, interconnect attributes, ILD thickness, and device characteristics, (ii) effects of electrostatic coupling for M3D circuits and corresponding models, and (iii) NoC parameters including number of cores, routers, links, and associated router connectivity. We quantify the effects of electrostatic coupling while evaluating the performance of the M3D NoC. Subsequently, we design the electrostatic couplingaware M3D NoC architecture and undertake a detailed performance evaluation. Latency, energy, and energy-delay-product (EDP) of the optimized M3D NoC are the final outputs. In this work, we focus on the trade-off between the performance benefit and electrostatic coupling effect in M3D 
NoC. In addition, we analyze the characteristics of routers and overall traffic patterns of the M3D NoC under electrostatic coupling effects. To the best of our knowledge, this is the first work that considers the effects of electrostatic coupling in designing M3D NoC architectures.

The rest of the article is organized as follows. Section 2 discusses related prior work. In Section 3, we describe the M3D-enabled NoC design space and the preliminaries of electrostatic coupling. Section 4 presents the formal problem setup and the proposed solution. The performance evaluation and analysis are described in Section 5. Finally, we summarize the key findings of this work in Section 6.

\section{RELATED PRIOR WORK}

To exploit the advantages of 3D integration, prior work has addressed the design, synthesis, and optimization of application-specific NoC architectures. The Sunfloor 3D is an example for developing application-specific 3D NoCs, which focused on minimizing the wirelength and the resultant capacitance to ensure high performance (Seiculescu et al. 2009). Later, a more general-purpose 3D NoC was proposed using an Integer Linear Programming (ILP)-based algorithm to insert longrange links to develop low diameter and low-radix architecture (Akturk and Ozturk 2013). These design and optimization approaches primarily focused on TSV-based 3D architectures and performance benefits offered by the monolithic integration are not fully studied.

The merits of M3D-based designs have also been explored (Lin et al. 2006; Bobba et al. 2013). The smaller dimensions of the ILD and MIV offer high-density integration, flexibility of partitioning logic blocks across multiple tiers, and significant reduction of total wire-length (Bobba et al. 2013). For M3D, the total parasitic capacitance and the number of buffers are also less for clock signals (Acharya et al. 2016). It was found that the footprint and wirelength of M3D-based designs are reduced by $42 \%$ and $23 \%$, respectively, over their planar counterparts at the $45 \mathrm{~nm}$ technology node (Lee et al. 2013). This results in a 37.5\% reduction in power consumption for M3D-designs for the same technology node. Moreover, the performance improves further for more advanced technology nodes such as 22, 14, 7nm, and so on (Lee et al. 2012; Acharya et al. 2016; Samal et al. 2016). The effects of the number of planar tiers, tier-level partitioning, and MIV insertion methodology on the performance of M3D-based ICs were analyzed in Kim et al. (2016b). It was found that as the number of MIVs inserted in the design increases, the power savings improve as well. Motivated by the promise of monolithic integrations, the CELONCELPD design framework was proposed to explore the merits of gate-level partitioning and cell-on-cell stacking design methodology for M3Dintegration (Bobba et al. 2013). The authors in Golshani et al. 2010; Batude et al. (2008) studied the performance of M3D-enabled SRAM and illustrated the advantages of M3D-based designs. Monolithically integrated 3D system enabled by nanotechnologies (N3XT) is proposed in the literature (Sabry et al. 2015). N3XT employs recent nanotechnologies such as carbon nanotubes and monolithic 3D integration and achieves high-performance and energy efficiency. The above-mentioned recent works on M3D principally focus on technology and generic circuit and system design, not on manycore chip design. Hence, our article complements the findings of the existing works, like N3XT (Sabry et al. 2015).

The authors in Das et al. (2017) explored the design space of irregular NoCs and demonstrated the efficacy of M3D integration (Das et al. 2017). However, all these studies primarily focused on the performance evaluations of M3D-designs without considering any reliability challenges that arise due to the presence of extremely thin inter-layer dielectric (ILD) between the tiers (Yu et al. 2016). Recently, the impact of electrostatic coupling and wafer-bonding on path delay of M3D has been analyzed (Koneru et al. 2017). The effect of electrostatic coupling starts to impact the timing characteristics of M3D IC when the ILD thickness is less than $100 \mathrm{~nm}$. The negative effects of electrostatic coupling become significant for ILD thickness smaller than 50nm. 
In this work, we characterize the impact of electrostatic coupling on the achievable performance of M3D NoC. We focus on the system-level design optimization to circumvent the impact of electrostatic coupling. Alternatively, we consider two opposing effects during the design and optimization of M3D-enabled system, e.g., the benefit of lower energy consumption of vertical interconnects enabled by the MIVs and the timing penalty introduced by electrostatic coupling. To achieve the optimum latency and energy consumption, we maximize the benefits offered by the M3D integration while minimizing the impact of electrostatic coupling during the system-level design optimization.

\section{DESIGN SPACE OF M3D-BASED NOC}

In this section, we introduce the design space of M3D-enabled NoC and discuss the effects of electrostatic coupling on the overall achievable performance. We consider the small-world networkenabled 3D NoC (3D SWNoC) as the testbed architecture. This particular architecture is chosen due to its superior performance over other existing regular and irregular counterparts in terms of latency and energy (Das et al. 2017).

\subsection{Performance and Energy Improvements in M3D NoC}

In an NoC, the routers and the associated links form the communication backbone. The primary focus of an efficient NoC is to ensure lower latency and energy consumption per message. Both network latency and energy consumption are affected by the characteristics of the underlying physical layer. We can exploit the benefits of M3D integration to reduce NoC latency and energy consumption. Overall, the NoC design space consists of cores, number and types of routers (number of router ports, single- vs. multi-tier), number of links and their lengths, the interconnection architecture, and placement of routers and links.

One of the primary advantages of M3D integration-based design over the traditional TSV-based counterpart is that it offers the flexibility of implementing logic blocks over multiple tiers. In M3Denabled NoC, the routers can be designed to extend over multiple tiers. The benefits of extending the routers over multiple tiers are threefold. First, the intra-router wirelength and the energy consumption are reduced. For example, if we extend a standard low-density parity check (LDPC) coder circuit to two-tiers from single-tier, then the wirelength reduces by $16 \%$ and energy efficiency improves by $34 \%$ (Kim et al. 2016b). The reduced wirelength offers lower delay in critical paths and increased operating frequency for the corresponding NoC. Second, the inter-router hop count of the NoC reduces as routers extended over multiple tiers can communicate with other routers without traversing planar links (Das et al. 2017). This reduction of hop count improves both the performance and energy savings of the M3D-based NoCs. Third, MIVs enable the vertical communication for M3D NoCs. In general, the resistance of the MIV (10 $\Omega$ for a $50 \mathrm{~nm}$ diameter) is significantly higher than that of a TSV $(0.05 \Omega$ for a $2 \mu$ m diameter) (Samal et al. 2016). However, the parasitic capacitance of MIVs $(0.2 \mathrm{fF}$ for a $50 \mathrm{~nm}$ diameter) is an order of magnitude ( $\sim 15$ times) lower than that of TSVs (3fF for a $2 \mu$ m diameter) (Samal et al. 2016). While the energy consumption of MIVs is comparable to that of planar metal wires, the associated energy per bit for the TSVs is much higher. Depending on the technology parameters, values of the typical TSV energy per bit are 4 to 5 times more than that of the planar metal wires (Samal et al. 2016; Nayak et al. 2015). The overall result of the above-mentioned benefits is that the M3D NoC is capable of outperforming TSV-based counterparts.

\subsection{Effects of Electrostatic Coupling in M3D NoC}

Electrostatic coupling is specifically pronounced when the separation between two neighboring circuits is reduced to the order of tens of nanometers (Yu et al. 2016; Batude et al. 2008). Figure 2 


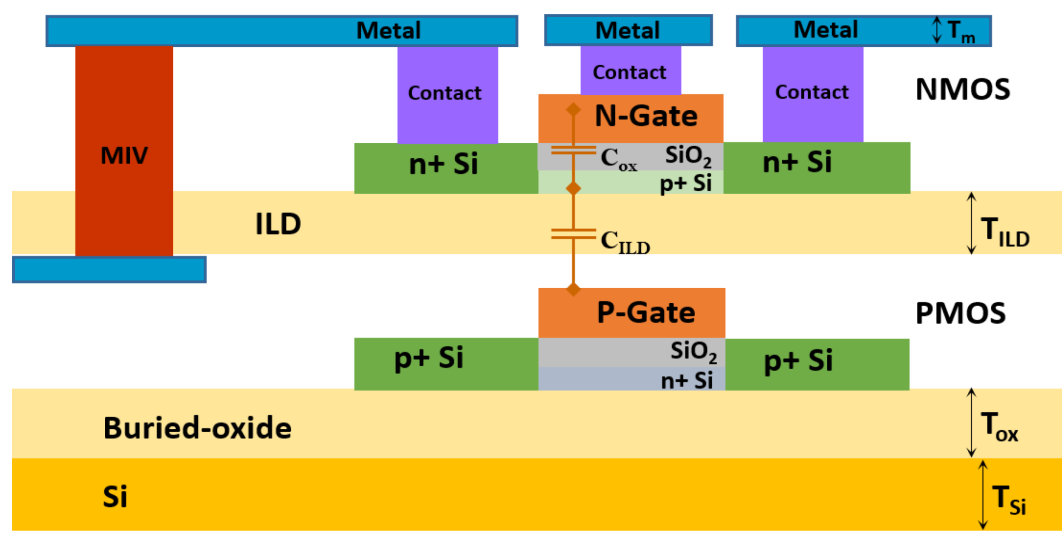

Fig. 2. Schematic of the simple capacitance network model in monolithic 3D-based IC on a fully depleted SOI MOSFET (Hiramoto et al. 2003; Yu et al. 2016). The size of the MIV ranges from 50 to $\sim 100 \mathrm{~nm}$. ILV refers to the inter-layer-dielectric and varies from 30 200nm. $T_{I L D}, T_{o x}, T_{s i}$, and $T_{m}$ are thickness of ILD, buried oxide, silicon and metal layer, respectively. $\mathrm{C}_{\text {ILD }}$ and $\mathrm{C}_{\mathrm{ox}}$ are the depleted film capacitance and gate oxide capacitance, respectively (Batude et al. 2017; Panth et al. 2014; Lee et al. 2013).

shows a cross section of the M3D-enabled two-tier 3D IC with different parameters affecting the electrostatic coupling between the PMOS and NMOS transistor from adjacent tiers. In this situation, the threshold- and switching-voltages of any victim transistor are affected by an aggressor. This, in turn, leads to variation in the transistor current and overall delay of the corresponding critical paths. For example, the threshold voltage, $V_{t h}$, of a PMOS transistor increases by $130 \mathrm{mV}$ when the gate voltage of a neighboring NMOS, located $10 \mathrm{~nm}$ from the PMOS, is switched from 0 to $1 \mathrm{~V}$ in $45 \mathrm{~nm}$ FDSOI technology (Batude et al. 2008). This coupling phenomenon can significantly alter the delay of the victim circuit. While a guard-band-based design can compensate such timing penalties, however, if the delay is more than the guard-band (in general $10 \%$ positive slack is employed (Jeong et al. 2009)), the design can violate the timing specification of the system leading to significant performance degradation.

In M3D-enabled NoCs, router architectures are typically optimized to reduce the intra-router wirelength and parasitic capacitance. Two types of router configurations are possible with M3D integration viz. single- and multi-tier routers. For multi-tier routers, if the ILD thickness is low enough, then router logic residing in a particular tier is affected by electrostatic coupling arising from the router logic in the other tier. This in turn gives rise to variation in the intra-router path delay. The NoC routers consist of multiple ports and the router logic belongs to each port. In this work, we consider the effects of the electrostatic coupling for intra-router communication for multi-tier routers. Hence, to capture the coupling effect in multi-tier routers, we determine the delay from the input port to output port including each stage in multi-tier routers. However, for single tier routers, there is no scope for intra-router electrostatic coupling. Moreover, during the placement and routing stages, we minimize the effects of electrostatic coupling for singletier routers by restricting the usage of upper layer metals in the bottom tiers. This effectively increases the distance between upper-tier transistor and the aggressor at the bottom-tier (more than 50nm does not have significant coupling effects (Yu et al. 2016)). This ensures that single tier-routers do not violate the timing constraints. However, we cannot employ such a strategy for multi-tier routers and hence, the electrostatic coupling is more pronounced for multi-tier routers than their single-tier counterparts. The variation in timing delay along the critical path affects the 
intra-router stage delay. This negatively affects the NoC performance in terms of both latency and energy overheads.

In addition, the traffic carrying capacity of the routers also plays an important role here. Large routers carry more traffic than small ones. Hence, when these large routers are extended over multiple tiers, then more NoC traffic would be affected by the electrostatic coupling induced delay. Hence, for large routers, the effects of electrostatic coupling will be more pronounced.

\section{OPTIMIZATION OF M3D NOC}

In this section, we first present how the intra-router delay is affected by the electrostatic coupling in M3D NoCs. Subsequently, to consider the effects of electrostatic coupling on the NoC performance, the intra- and inter-router delays need to be formulated by incorporating the device parameters of M3D integration.

\subsection{Delay Modeling of Electrostatic Coupling}

The performance of an M3D NoC, in the presence of electrostatic coupling, depends on two factors: (i) performance and energy improvements achieved from the multi-tier routers enabled by MIVs; (ii) the latency penalty introduced by the electrostatic coupling. Considering these two factors, our aim is to determine an optimized M3D NoC configuration that maximizes the performance by minimizing the effects of electrostatic coupling. More specifically, we develop a design methodology that determines the number and placement of multi-tier routers of an M3D-enabled NoC. Alternatively, the output of the proposed methodology is to determine which particular router should be extended over multiple tiers and how they should be placed to communicate with other routers more efficiently for improved performance and energy-efficiency.

The relation of the electrostatic coupling between device layers in M3D ICs was analyzed by Koneru et al. (2017). They also carried out a detailed device simulation to understand the impact of coupling on the threshold voltage of a top-layer transistor for both transistor- and gate-level integration. By adopting a conventional SOI transistor to transistor level integration as well as gate-level partitioning, for both N-channel and P-channel double-gate transistors, the $\left|V_{t h}\right|$ was obtained to be $51 \mathrm{mV}$ for an ILD thickness of $23 \mathrm{~nm}$. The variation in threshold voltage is reduced as the ILD thickness is increased. In addition, the body-to-gate voltage has significant impact as well. The findings of this work (Koneru et al. 2017) demonstrated that for ILD thickness greater than $100 \mathrm{~nm}$, coupling between the top and bottom layers is minimal. For ILD thickness less than $50 \mathrm{~nm}$, the impact of coupling on threshold voltage of a top-layer transistor is significant and cannot be ignored. The findings of the above-mentioned work have been a key motivation for us to study the impact of electrostatic coupling for the M3D NoC. Instead of depending on empirical rulebased margin, we considered the variation in $\left|V_{t h}\right|$ obtained from the above-mentioned reference to design the optimum M3D NoC architecture.

In an M3D-enabled design, the threshold voltage of transistors in multi-tier routers is affected by the presence of electrostatic coupling between two-adjacent tiers. As the thickness of interlayer-dielectric (ILD) layer decreases, the threshold voltage increases in the fully depleted FDSOI technology. This phenomenon is similar to the classical coupling relation of asymmetric doublegate fully depleted (FD) silicon on insulator (SOI) MOSFET caused by the back-gate voltage and depleted capacitance between channel and substrate (Hiramoto et al. 2003). The variation in threshold voltage is described by Ernst et al. (2003) and Hiramoto et al. (2003),

$$
\Delta V_{t h} \approx\left(\frac{C_{I L D}}{C_{O X}}\right) \Delta V_{g s} \approx\left(\frac{T_{O X}}{T_{I L D}}\right)\left(\frac{\varepsilon_{I L D}}{\varepsilon_{O X}}\right) \Delta V_{g s},
$$


where $T_{O X}$ and $T_{I L D}$ are the thickness of gate-oxide layer and ILD, respectively; the parameters $\varepsilon_{I L D}$ and $\varepsilon_{O X}$ are the dielectric constants of oxide and silicon. The parameter $\Delta V_{g s}$ represents the variation of the gate voltage of the bottom MOSFET. In the above equation, $C_{I L D}=\varepsilon_{I L D} / T_{I L D}$ is the depleted-film capacitance, and $C_{O X}=\varepsilon_{O X} / T_{O X}$ is the gate-oxide capacitance. As the ILD thickness decreases, the body effect factor $\left(C_{I L D} / C_{O X}\right)$ increases. Hence, the threshold voltage increases. To explain the above relations, Figure 2 shows the schematic of the simple capacitance model and layer structures of M3D.

The variation of threshold voltage impacts the timing characteristics of logic blocks from adjacent tiers. As the threshold voltage $\left(V_{t h}\right)$ increases, the delay of a gate with electrostatic coupling $\left(t_{\text {coupling }}\right)$ increases as well. To capture the effects of variation of threshold voltage on the gate delay, $\left(t_{\text {coupling }}\right)$, we adopt the following model (Rabaey et al. 2003; Yu et al. 2016):

$$
t_{\text {coupling }} \approx \frac{C_{L} V_{D D}}{\beta_{n}\left(V_{D D}-V_{t h}\right)^{2}},
$$

where $\beta_{n}$ is the transistor size-dependent parameters, and $C_{L}$ is output load capacitance. Having an inverse squared relationship with threshold voltage $\left(V_{t h}\right)$, the delay, $t_{\text {coupling }}$, increases significantly when ILD thickness is smaller than $50 \mathrm{~nm}$ (Yu et al. 2016).

Finally, the intra-router delay $(T)$ can be expressed as a function of FO4, as

$$
T=f(\mathrm{FO} 4) \text {. }
$$

In the above equation, the delay of each pipelined stage of the NoC routers can be expressed in terms of FO4 (Grecu et al. 2004; Peh and Dally 2001). The delay of a gate is affected by coupling, which in turn changes the value of FO4 delay. For each router, we determine the delay of each pipelined stage in term of FO4 without and with the coupling effect. For multi-tier routers, the intra-router delay will be changed depending on the ILD thickness and coupling induced gate delay, $t_{\text {coupling. }}$ To achieve the minimum NoC latency, this intra-router delay model of Equation (3) is employed to capture the electrostatic coupling effects.

\subsection{Problem Formulation}

We consider a NoC design space that consists of $N$ number of cores and routers arranged in $L$ number of tiers. In the M3D NoC, each core is associated with one router and hence, the number of routers is $N$. The routers can be placed in a single tier or extended over multiple tiers. The interrouter communication frequency and Cartesian distance between $i$ th and $j$ th routers are denoted by $f_{i j}$ and $d_{i j}$, respectively. To optimize the placement of the multi-tier routers and associated links to achieve the minimum NoC latency, we define $C$ as the communication cost and target to achieve its near-optimal value. The communication cost is the product of frequency of communication, router, and link delays summed over every source and destination pair, i.e.,

$$
\begin{aligned}
& C=\sum_{i=1}^{N} \sum_{j=1}^{N}\left(\sum_{k=1}^{h_{i j}-m_{i j}(n)} T_{i j, k}+d_{i j} \cdot \tau\right) \cdot f_{i j}, \\
& m_{i j}(n)\left\{\begin{array}{l}
=0, \text { if } n=0 \\
>0, \text { if } n>0,
\end{array}\right.
\end{aligned}
$$

where, $h_{i j}$ is the hop count between the $i$ th and $j$ th routers, and $m_{i j}(n)$ denotes the reduction in hop count due to the presence of $n$th multi-tier router between the ith and the $j$ th router. If a multitier router is placed on the path between two communicating routers, then the inter-router hop count of the network reduces as the routers extended over multiple tiers can communicate with other routers without traversing planar wires. The parameter, $T_{i j, k}$, refers to the intra-router delay 
of the $k$ th router Equation (3) between $i$ th and $j$ th routers. The physical distance between $i$ th and $j$ th router is denoted by the parameter, $d_{i j}$. The total delay of an interconnect having a length of $d_{i j}$ depends on the delay of the wire and the associated buffers along the path. The parameter, $\tau$, refers to delay of the buffer and wire per unit length. In the presence of electrostatic coupling, the multi-tier routers experience additional delay and that in turn increases the value of $T_{i j, k}$, which is determined by Equation (3) in the previous section. Optimizing the cost function, $C$, ensures lower average hop count and improves the overall NoC latency.

The energy consumption in an NoC consists of the energy dissipated by both links and routers, i.e.,

$$
E=\left(\sum_{i=1}^{L} E_{\text {Link }, i}+\sum_{j=1}^{N} E_{\text {Router }, j}\right),
$$

where $E_{\text {Link }}$ and $E_{\text {Router }}$ refer to the energy consumed by the links and routers, respectively; $L$ is the total number of links in the NoC. For each router, the total energy consumption depends on the router port count, number of intra-router stages, and the amount of traffic passing through it. For multi-tier routers, the energy consumption decreases due to reduction of effective wirelength. However, in the presence of electrostatic coupling, the multi-tier routers experience additional delay, which increases the energy consumption. However, the link energy depends on the link length and the amount of traffic passing through it. In an M3D NoC, energy-efficient MIVs help in reducing the overall link energy.

The objective of NoC design optimization problem is to find the best NoC design $\left(d^{*}\right)$ from the feasible design space $(D)$ subject to various design constraints. Here, the best NoC design, $d^{*}$, refers to the NoC configuration having near optimal values of the cost functions $\mathrm{E}$ and/or $\mathrm{C}$. To ensure the candidate M3D NoC design is physically plausible and energy efficient, we consider the following constraints: (i) Connectivity constraint: the NoC must be a connected graph (this ensures every source and destination can communicate with each other). (ii) Router port constraint: the maximum number of ports $\left(k_{\max }\right)$ should be less than an allowable upper limit so that none of the routers become too large (Kim et al. 2016a). This constraint is considered due to the fact that big routers with large number of ports consume more energy than an average-sized router. It has been shown that for a small-world network-enabled 3D SWNoC, this number is 8 (Kim et al. 2016a). (iii) Link constraint: the number of links in a 3D SWNoC $\left(L_{3 D} S W N_{0} C\right)$ is equal to that of a 3D MESH NoC $\left(L_{3 D} M E S H\right)$ of the same system size. This constraint ensures that the 3D SWNoC does not introduce any additional link overhead. More specifically,

$$
\begin{aligned}
\text { Inputs : } & f_{i j}, N, L, \\
\text { Outputs : } & \text { Best M3D NoC design, } d^{*}, \\
\text { Objectives : } & d^{*}=\arg \min _{d \in D}\{O\}, \\
& \text { where, } O=\{E \text { or } C \text { or }(E, C)\}, \\
\text { Subject to : } & \text { fully connected } S W \text {-graph, } \\
& k_{\text {max }}<k_{\text {max }, \text { allowed }}, \\
& L_{3 D S W N o C}=L_{3 D M E S H} .
\end{aligned}
$$

Here, $N$ and $L$ refer to the total number of routers and links in M3D NoC design space as defined above. The objective function, $O$, changes depending on the target optimization problem. For single objective optimization, it is either $E$ or $C$, which targets to explore the design space to find the M3D NoC with lowest energy $(E)$ or latency $(C)$, respectively. Achieving the lowest NoC latency for the NoC may not always ensure the best energy consumption profile and vice versa. Hence, to reduce both NoC latency and energy consumption simultaneously, we employ a multi-objective 


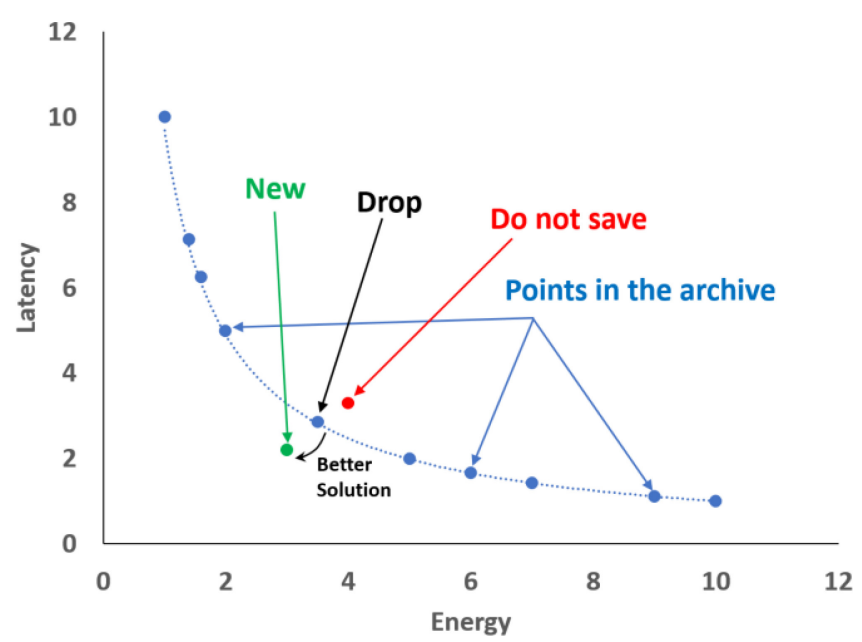

Fig. 3. Illustration of archive solutions in AMOSA. The blue points are the current solutions in the archive. If a new solution is created, then we decide to add or drop in the archive based on the current solution.

optimization (MOO)-based approach to explore the design space of M3D-enabled NoCs. For this case, $O$ includes two objectives $E$ and $C$ simultaneously.

In $\mathrm{MOO}$, the main challenge is to identify the candidate solution that performs equally well for both objective functions $E$ and $C$. Using the straightforward simulated annealing approach to explore the NoC design space is computationally expensive and time consuming (Bandyopadhyay et al. 2008). In this context, we employ the well-known AMOSA framework that has been found to minimize the runtime of $\mathrm{MOO}$-problems significantly while ensuring high-quality solutions (Bandyopadhyay et al. 2008). In AMOSA, an archive is created to collect the non-dominating and best quality NoC solutions obtained by optimizing different objective functions. In AMOSA, archived solutions are a set of NoC designs with different trade-offs. To explain the AMOSA framework adopted for $\mathrm{NoC}$ optimization and the role of archived solutions, Figure 3 shows the process of selecting new candidates from the archived solution set. The horizontal axis refers to the energy metric while the vertical axis refers to the latency per message (two different objective functions are shown in the two orthogonal axes).

In Figure 3, the blue points are archived solutions, one of which can be the potential final solution at the end of optimization. During the optimization, if an inferior solution compared to the archived candidate (the red solution) is created, we do not save the solution in red, which is worse than the solutions in the archive. If a better solution (corresponding to the green dot in the figure) is created, then we save the solution in the archive while dropping one of the other worse solutions from the archive.

During each optimization step, one of the archived solutions is perturbed to generate a new configuration. As we focus on router and link perturbation in the M3D-NoC architecture design space, we utilize two moves for solution perturbation namely the new link placement and the router configuration change as follows:

- New link Placement-L(l): Select and remove a link of length- $l$ between two randomly selected routers.

- Router configuration change $-R(r)$ : Select a router and choose a single or multi-tier router. If a router is on the bottom tier, then the router is extended over top tier. If a router is on the top tier, then the router is extended over bottom tier. 
The AMOSA algorithm is built on the principle of simulated annealing (SA). If the objective function (as described in Equation (8)) is denoted by $O$, then we first calculate difference in objective function value, $\Delta O$, at a given temperature $(T)$. Subsequently, we determine the acceptance of the new architecture using Boltzmann distribution (Kirpatrick et al. 1983; Bandyopadhyay et al. 2008). A new state $s$ is designated with Equation (12).

$$
p_{q, s}=\frac{1}{1+e^{-(O(q)-O(s)) / T}},
$$

where $q$ is the current state and $O(q)$ and $O(s)$ are the corresponding cost of $s$ and $q$, respectively. We choose large enough values for temperature and cooling rate to avoid local optimum. This process continues until the temperature is lower than 1. Based on the quality of new solution, the archive is updated. Thus, on completion of executing the AMOSA algorithm, we obtain a set of archived NoC configurations that correspond to the Pareto front. Among these candidate NoC designs, we choose the configuration with the lowest latency, energy, and/or EDP (depending on the target objective function) as the final solution.

To quantify the vast design space of M3D-enabled NoC, we consider the traditional TSV-based NoC configuration as a baseline. In M3D-enabled NoCs, the cores and routers are extended over multiple tiers and the corresponding NoC optimization space is much larger than the TSV-based counterparts, where cores and routers are only placed in planar layers. For example, the size of the candidate set for the location of links is (N/P)! and (N)! in TSV- and M3D-based designs, respectively, where $\mathrm{N}$ is the number of cores and $\mathrm{P}(>1)$ is the number of planar layers in TSV-based design. For a high number of cores $(\sim 1,000)$ and a finite number of planar layers in TSV-based 3D IC, the difference in the sizes of candidate link location sets is enormous. It can be easily shown that the search space is at least $P^{N}$ times larger for M3D, e.g., an intimidating $3^{1,000}$ times for $\mathrm{N}=$ 1,000 and $\mathrm{P}=3$. Moreover, for a given MIV budget $\mathrm{B}$, we need to search over all B-size subsets of link locations, which further increases the complexity of the search problem. To handle such an optimization problem, we have incorporated the SA-based approaches as it has been popularly used in numerous previous works in the EDA field. For single-objective optimization, we use SA while for multi-objective we employ the well-known AMOSA algorithm (Bandyopadhyay et al. 2008).

\section{EXPERIMENTAL RESULTS AND ANALYSIS}

In this section, we present a detailed performance evaluation of the M3D NoC in the presence of electrostatic coupling and evaluate the efficacy of coupling-aware design optimization to improve the M3D NoC performance (M3D NoC refers to the M3D-enabled SWNoC as described in Section 3).

\subsection{Experimental Setup}

To evaluate the performance of M3D NoC, we consider a system with 64 cores and 64 routers equally partitioned in two tiers with each tier having an area of $200 \mathrm{~mm}^{2}$. In each tier, 32 cores are placed at regular intervals in a grid pattern. We employ wormhole routing for data exchange. Due to irregular nature of the 3D SWNoC architecture, the topology-agnostic Adaptive Layered Shortest Path Routing (ALASH) is employed for data exchange (Lysne et al. 2006). The NoC routers were synthesized using Synopsys Design Vision. Energy consumption of the wireline links was obtained through HSPICE simulations, taking into consideration the length of the wireline links. It should be noted that as the MIVs are as small as local vias in 2D library, hence during synthesis, we do not need to add any additional overhead (Lee et al. 2013; Nayak et al. 2015). To obtain the detailed processor and network level information, we use GEM5 (Binkert et al. 2011), the full system mode, 
for a system consisting of $64 \times 86$. We use Ruby memory model with MOESI_CMP_directory protocol. It consists of $64 \mathrm{~KB} \mathrm{L1}$ (data + instruction) and a shared 8MB L2 cache. For performance evaluation of different M3D NoCs, we consider four SPLASH-2 benchmarks (FFT, RADIX, LU, and WATER) (Woo et al. 1995) and four PARSEC benchmarks (DEDUP, VIPS, FLUIDANIMATE (FLUID), and CANNEAL (CAN)) (Bienia 2011). These benchmarks are chosen as they widely vary in communication and computation characteristics.

\subsection{Effect of Electrostatic Coupling on M3D NoC Performance}

In this section, we evaluate the effects of electrostatic coupling on the performance of M3D NoC. It has been reported that it is possible to fabricate an M3D IC with ILD thickness as low as 23nm (Batude et al. 2011; Batude et al. 2012). These devices were characterized and measurement data were reported. Such extreme scaling of ILD thickness enables the integration of more transistors per unit area. However, as shown in Koneru et al. (2017), such extreme scaling of ILD thickness leads to electrostatic coupling. When M3D is eventually commercialized and utilized for NoC design, an appropriate value of the ILD thickness will be selected based on a cost/benefit analysis. In this work, we have presented the impact of electrostatic coupling by varying the ILD thickness. We have experimentally investigated the trade-off between the performance benefits and electrostatic coupling effect in M3D NoC.

To describe the effects of electrostatic coupling on the M3D NoC, we consider the ILD thickness to be $50 \mathrm{~nm}$ as a case study. To evaluate electrostatic coupling effects, we consider latency, energy, and energy-delay-product (EDP) as three relevant performance metrics. The EDP is defined as the product of the NoC latency and energy per message. To achieve the lowest latency and the lowest energy, we employ the single objective optimization approach described in Section 4.2 and consider objective functions $C$ and $E$ (Equations (4) and (6)), respectively. To explore the NoC design configuration with the lowest EDP, we consider the MOO approach where both the $E$ and $C$ objectives are optimized simultaneously. To study the effects of electrostatic coupling, we consider three cases: (i) the NoC is optimized to have the lowest latency without considering the effects of electrostatic coupling (this is an instance of electrostatic unaware design). As shown in Das et al. (2017), for the electrostatic unaware design, almost all the routers become multi-tier. This is our baseline scenario. (ii) We incorporate the effects of electrostatic coupling in to the baseline configuration and determine its effects on latency, energy, and EDP of the NoC. (iii) We consider the effects of electrostatic coupling during the NoC design and optimization stages. Subsequently, we determine the numbers and locations of the multi-tier routers that minimize the negative effects of electrostatic coupling on the performance of the M3D NoC.

Figures 4(a)-4(c) show the normalized latency, energy, and EDP of the M3D SWNoC for the above mentioned three cases. They are denoted as the baseline, baseline with electrostatic coupling (w/coupling), and coupling-aware, respectively. All the performance metrics are normalized with respect to that for the baseline. It is evident that the baseline w/coupling shows, on an average, $7.3 \%, 10.1 \%$, and $18.1 \%$ increase in the latency, energy, and EDP, respectively, compared to the baseline. This implies that the electrostatic-coupling influences the achievable performance noticeably. Hence, for an M3D NoC, we should consider the electrostatic coupling during the design and optimization stages. When we consider the effects of the electrostatic coupling during the design and optimization stages, then the performance of the optimized M3D NoC improves (latency, energy, and EDP values are reduced) compared to the baseline w/coupling. On average, coupling-aware designs improve the latency, energy, and EDP by $3.7 \%, 4.1 \%$, and $12.5 \%$ compared to the baseline w/coupling counterparts. This happens due to two factors. First, for the coupling-aware design, the placement of routers and links is optimized to negate the effects of electrostatic-coupling. Second, the number of multi-tier routers are determined judiciously (detailed explanation is provided 


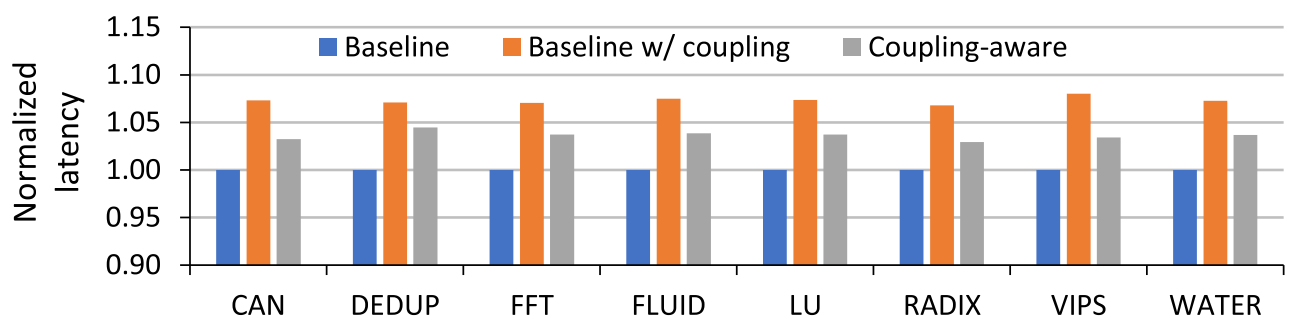

(a) Latency of the M3D NoC

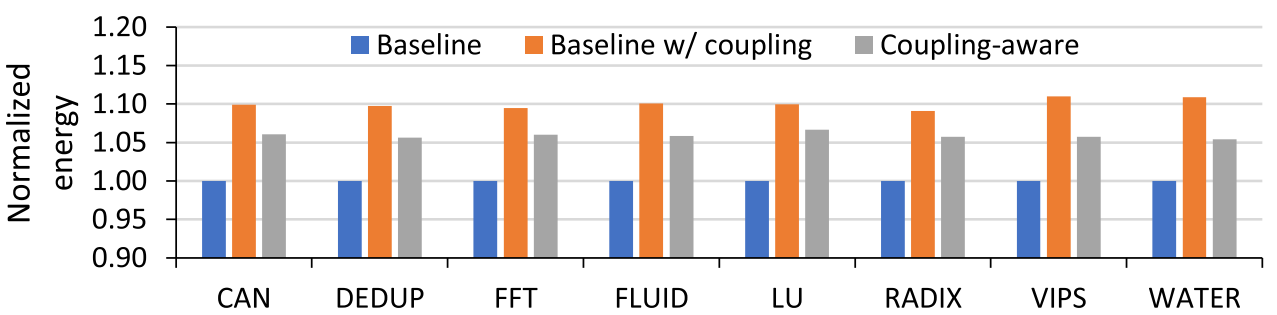

(b) Energy of the M3D NoC

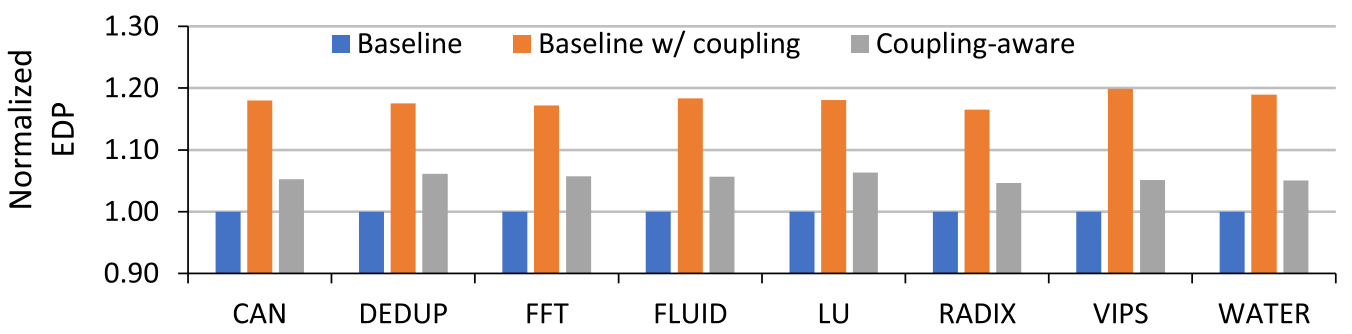

(c) EDP of the M3D NoC

Fig. 4. Effect of electrostatic-coupling on the (a) latency (optimized for latency only), (b) energy (optimized for energy only), and (c) EDP (multi-objective optimization) of the M3D NoC.

in the next subsection) to minimize the effects of electrostatic coupling. Hence, coupling-aware M3D NoC shows better latency, energy, and EDP than the baseline w/coupling. It is to be noted that by utilizing only a limited number of multi-tier routers (the numbers are explained in the next section) efficiently, we achieve similar performance of an M3D NoC that has almost all the routers multi-tier.

Next, we present the latency and energy consumption of the M3D SWNoC separately when it is optimized for EDP. This captures the effect of multi-objective optimization on each performance metric individually. In Figure 5(a), the latency of EDP optimized M3D SWNoC is normalized with respect to the baseline of latency optimized M3D NoC of Figure 4(a). The average latency values of the EDP optimized M3D SWNoC for baseline, baseline w/coupling and coupling-aware are higher than those latency optimized designs by $0.8 \%, 0.9 \%$, and $1.2 \%$, respectively. In Figure $5(\mathrm{~b})$, the energy of EDP optimized M3D SWNoC is normalized with respect to the baseline of energy optimized M3D NoC of Figure 4(b). The average energy values of the EDP optimized M3D NoC for baseline, baseline w/coupling and coupling-aware are higher than those energy optimized designs by $1.4 \%$, $1.7 \%$, and $2.3 \%$, respectively. By comparing both the Figures 4 and 5, one can observe that the multi-objective optimized M3D NoC incurs $1.2 \%$ latency and $2.3 \%$ energy penalty compared to 


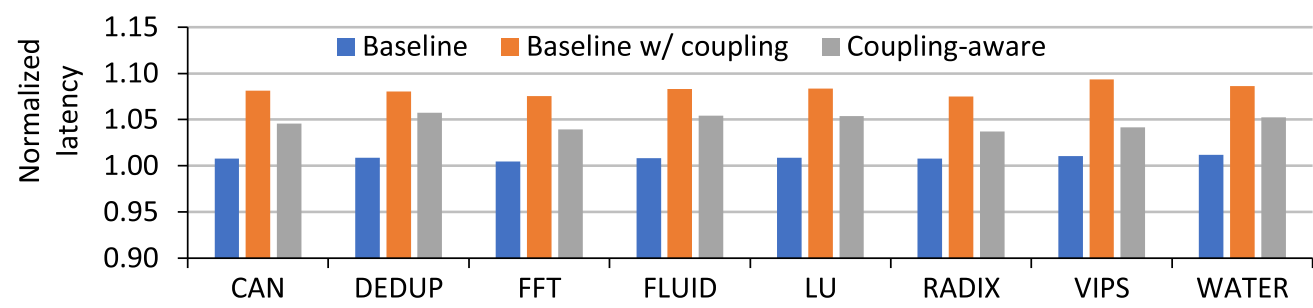

(a) Latency of the M3D NoC

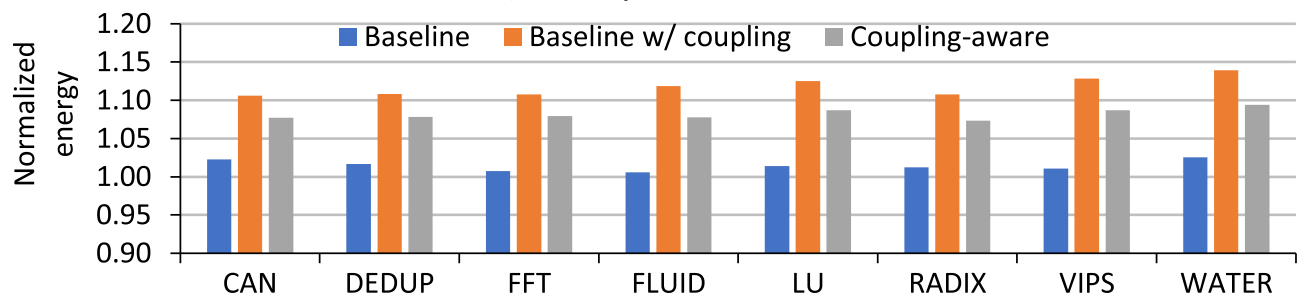

(b) Energy of the M3D NoC

Fig. 5. Effect of electrostatic-coupling on the (a) latency and (b) energy of the EDP optimized M3D NoC using AMOSA (normalized w.r.t. the baseline of latency and energy in Fig. 4).

the corresponding single objective optimization. However, when we optimize both latency and energy together (multi-objective optimization) then we achieve suitable balance between these two performance metrics and the overall EDP is minimized.

For M3D enabled designs, similar performance trends are observed for other NoC architectures as well. For example, to complement above mentioned analysis for the electrostatic coupling aware M3D SWNoC design and performance evaluation, we consider a M3D-enabled MESH NoC architecture where all the links are of same length. Figures 6(a)-6(c) shows the latency, energy per message and EDP for the baseline, baseline with electrostatic coupling and coupling-aware for different SPLASH-2 and PARSEC benchmarks for M3D-MESH NoC. As seen from this figure, the latency, energy and EDP of the baseline 3D MESH NoC degrades by $9.1 \%, 10.7 \%$, and $20.9 \%$, respectively. On average, coupling-aware designs improve the latency, energy and EDP by $4.4 \%, 6.3 \%$, and $11.2 \%$, respectively. Hence, we can conclude that the electrostatic coupling has similar effects on the performance of different types of M3D NoCs. This shows the efficacy of electrostatic coupling aware NoC design and optimization approach, which is applicable for any NoC architecture.

For latency-optimized and energy-optimized NoCs, the corresponding architectures achieve the best network latency and energy, respectively. However, these NoC configurations have higher EDP values compared to the EDP-optimized designs. The latency- and energy-optimized NoCs result in $6.8 \%$ and $2.7 \%$ higher EDP, respectively, than the EDP-optimized counterpart.

\subsection{Multi-tier Router Configuration}

In this section, we investigate the characteristics of multi-tier routers and overall traffic carrying patterns of the M3D NoC under electrostatic coupling effects. We also analyze the impact of NoC optimization on router connectivity and port distribution. As before the SWNoC is the testbed here too. We use the results in Section 5.2, which consider the 50nm ILD thickness as a case study.

In an $\mathrm{M} 3 \mathrm{D}$ NoC, the multi-tier routers contribute significantly to improve the NoC performance due to reduced hop count and interconnect length. However, they also experience the electrostatic coupling-induced performance degradations. Hence, we need to establish the trade-off between the NoC performance and the number of multi-tier routers. To illustrate this, Figure 7(a) shows 


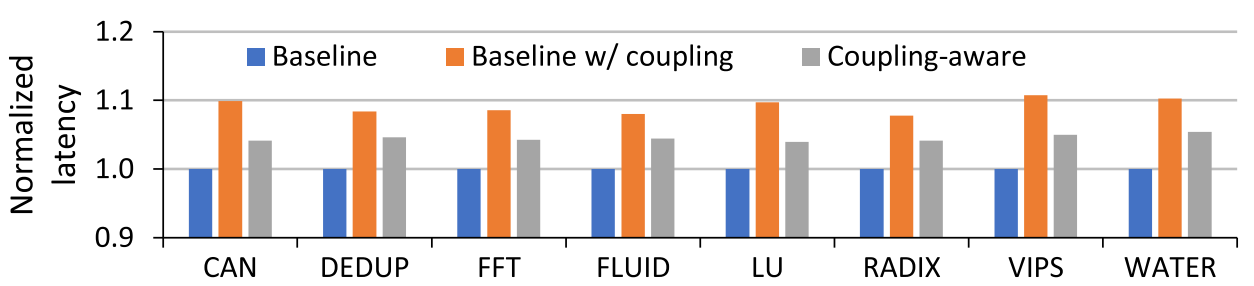

(a) Latency of the MESH NoC

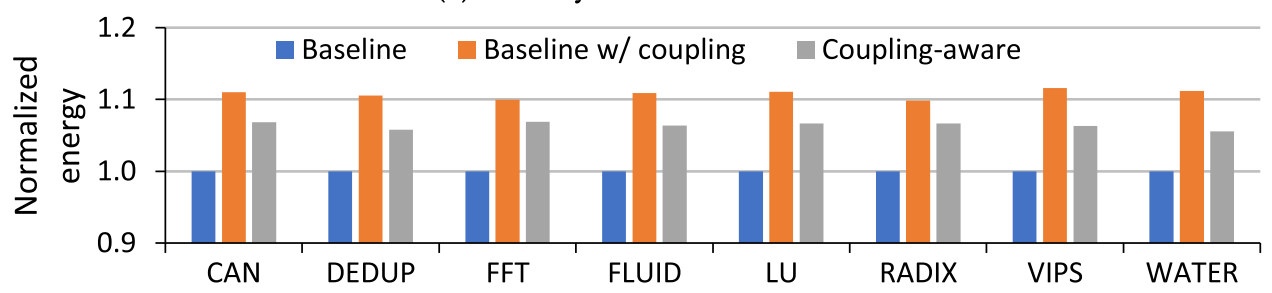

(b) Energy of the MESH NoC

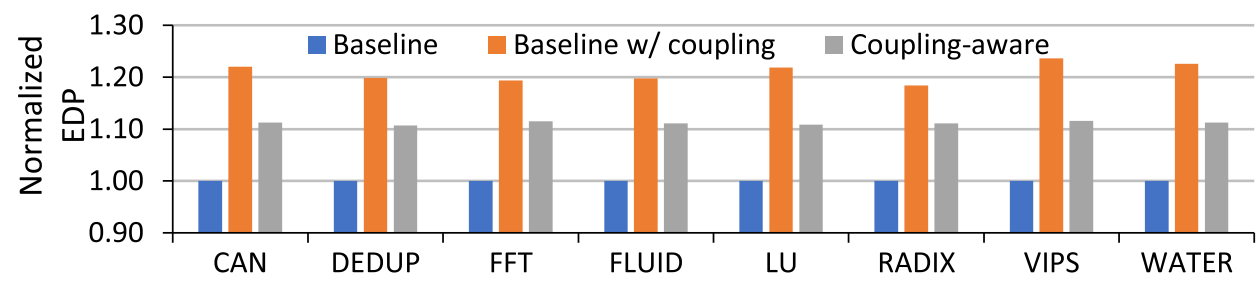

(c) EDP of the MESH NoC

Fig. 6. Effect of electrostatic-coupling on the (a) latency, (b) energy, and (c) EDP of the EDP optimized M3D MESH NoC.

the total number of multi-tier routers in the optimized M3D NoC for baseline w/coupling and coupling-aware approaches for each benchmark considered in this work. To simplify, Figure 7(b) shows the same number of multi-tier routers averaged over all benchmarks. As expected, the baseline w/coupling $\mathrm{NoC}$ has higher number multi-tier routers than the coupling-aware counterparts. The coupling-aware NoC lowers the number of multi-tier routers by $76.3 \%, 78.3 \%$, and $86.6 \%$ when compared to the baseline w/coupling for latency, energy, and EDP optimizations, respectively. In the coupling-aware design methodology, the number of multi-tier routers is selected by considering both the benefits of reduced hop-count and interconnect length and coupling-induced performance degradation. Hence, only a limited number of routers can be made multi-tier to achieve the performance benefit while incurring minimum penalty due to electrostatic coupling.

To explain the characteristics of the multi-tier routers further, we also analyze the overall NoC traffic patterns of the baseline w/coupling and coupling-aware M3D NoCs. We categorize the overall NoC traffic into two parts viz. planar and vertical traffic. The planar traffic consists of messages that stay on the same tier (horizontal plane) after passing through a router whereas the vertical traffic moves in between multiple tiers. In addition, there are two vertical traffic moves: vertical traffic between single-tier routers and intra-router vertical traffic in multi-tier routers. Figure 8 shows an illustrative example of both planar and vertical traffic in single- and multi-tier routers.

Figure 8 shows the traffic pattern of single- and multi-tier routers for the baseline w/coupling and coupling-aware M3D SWNoC for the WATER benchmark as a case study. The planar and 


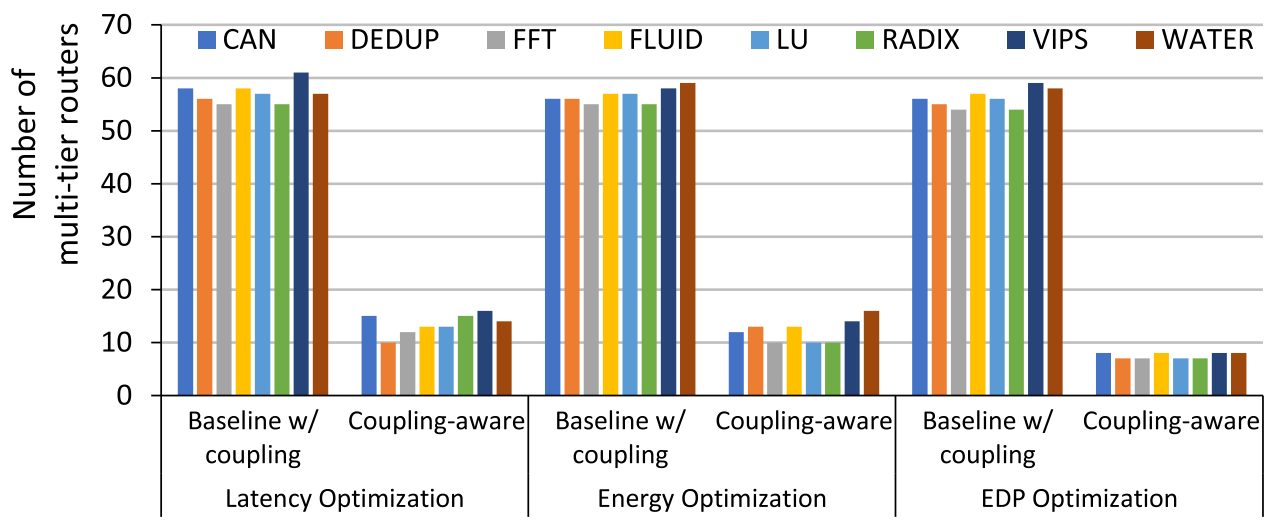

Fig. 7 (a). Number of multi-tier routers baseline with electrostatic coupling and coupling-aware for each benchmark.

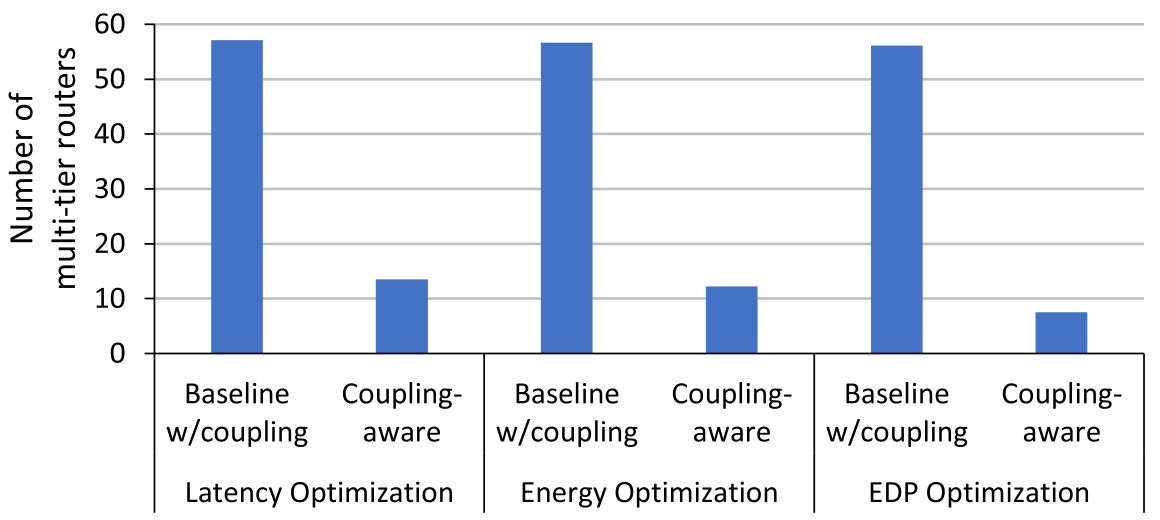

Fig. 7 (b). Number of multi-tier routers (averaged over all benchmarks considered in this work) with and without considering electrostatic coupling during optimization.
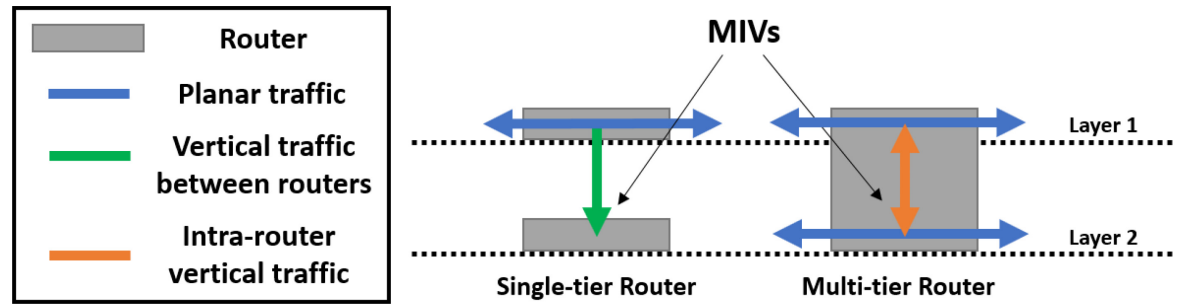

Fig. 8. Illustration of planar and vertical traffic for single- and multi-tier routers to analyze the electrostatic coupling effects.

vertical traffics are expressed as the percentage of the overall traffic. From Figure 9, we can observe that in the baseline w/coupling M3D SWNoC, the multi-tier routers carry more horizontal and vertical traffic than the single-tier routers. The reason is that the baseline w/coupling M3D SWNoC does not consider the coupling effect during design and optimization and use a large number of multi-tier routers. This ensures higher utilization of MIVs that are more energy efficient than the planar links. This also minimizes the average hop count of NoC. Hence, both link and router 


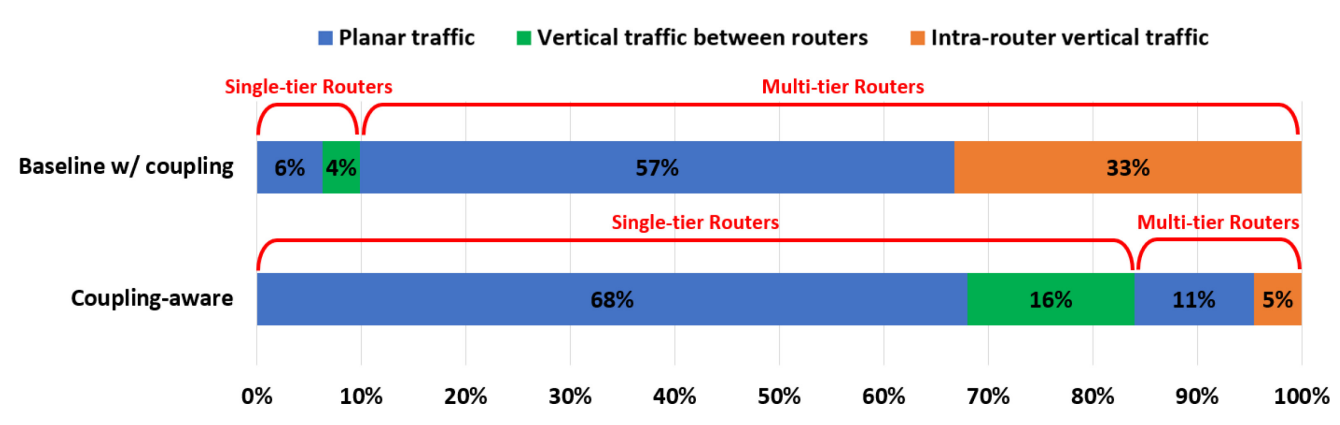

Fig. 9. Planar and vertical traffic distribution of single- and multi-tier routers with electrostatic couplingaware and -unaware optimization for WATER benchmark.

Table 1. The Power Consumption of Different Router Connectivity

\begin{tabular}{|c|c|c|c|c|c|c|}
\hline Router Connectivity & 3 & 4 & 5 & 6 & 7 & 8 \\
\hline \hline Power $(\mathrm{mW})$ & 2.51 & 3.36 & 4.21 & 5.06 & 9.41 & 10.80 \\
\hline
\end{tabular}

energies are reduced. However, in the presence of electrostatic coupling, the energy efficiency of MIVs is negated by the increase in delay and energy consumption of the multi-tier routers. Consequently, for the coupling-aware M3D SWNoC, the percentage of planar and vertical traffics for the multi-tier routers is reduced. From Figure 9, we can observe that in the baseline w/coupling M3D SWNoC, the multi-tier routers carry $90 \%$ of the total traffic. As the effects of electrostatic coupling are predominantly seen for multi-tier routers, hence, without any kind of coupling aware optimization, $90 \%$ of the traffic undergoes negative effects electrostatic coupling. At the same time, the multi-tier routers reduce the average hop count of the NoC (and thereby improves the NoC performance), and for a 64-core system the average hop count reduces by $8 \%$ (Das et al. 2017). For the coupling aware optimization, NoC routers are chosen selectively to achieve the average hop count advantage, while minimizing the negative effects for $90 \%$ of the total traffic. As a result, for coupling aware optimized $\mathrm{NoC}$, the number of multi-tier routers are reduced drastically. The total traffic passing through the multi-tier routers is lowered to $16 \%$ resulting in a reduction by $\sim 5$ times compared to the initial configuration. This additional traffic for coupling-aware NoC is carried by the single-tier routers (this minimizes the performance penalty incurred by the electrostatic coupling).

The variation in NoC performance is also related to the router connectivity. For example, Table 1 shows the power consumption of routers with varying size. Large routers (number of ports is more than the average) dissipate more energy than smaller routers. To explain the relation between the router connectivity and NoC performance, Figure 10 shows the router port distribution (total number of routers that has a particular port count) for the coupling-aware M3D NoC. This plot also shows the standard deviation of port counts of the routers. The NoC optimized to achieve minimum latency has a relatively flat port distribution curve with a standard deviation of 2.54. In contrast, the optimized NoC targeted to reduce energy consumption shows a highly skewed router-port distribution centralized around the average port count (with a standard deviation of $0.72)$. In this case, the number of routers having large port counts $(7,8$, and 9$)$ is reduced noticeably. The EDP-optimized M3D NoC balances both latency and energy and shows a port distribution curve that lies in between the other two distributions. This ensures that the EDP-optimized M3D NoC achieves latency improvement compared to multi-tier routers while minimizing the energy consumption caused by the big routers. 


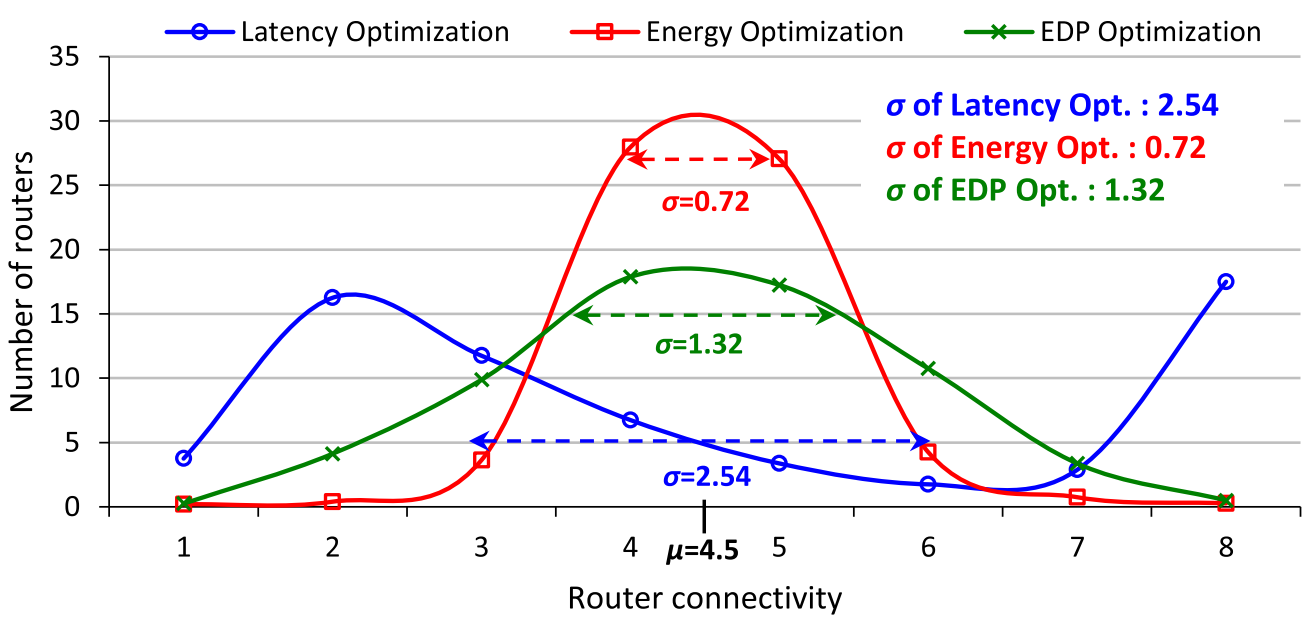

Fig. 10. Distribution of router-port counts for single- and multi-objective optimization approaches.

\subsection{Coupling-aware Optimization and ILD Thickness}

Low value of the ILD thickness and miniscule size of the MIV enable high-density integration, flexibility of partitioning logic blocks across multiple tiers, and offer significant reduction of total wire-length for M3D-enabled systems. However, the aggressive scaling of ILD thickness leads to a substantial variation in the threshold voltage of transistors (as shown in Equation (1) above). This variation of threshold voltage impacts the timing characteristics of logic blocks from adjacent tiers (as described in Equation (2)) and finally increases intra-router delay from the ideal case (explained in Equation (3)).

In this section, we compare the performance of the electrostatic coupling-aware and -unaware optimization of M3D designs with varying ILD thickness. We consider a wide range of ILD thickness to investigate the effect of electrostatic coupling and study five different cases: 20, 50, 100, and $150 \mathrm{~nm}$ ILD thickness. If the ILD thickness is increased beyond $150 \mathrm{~nm}$, then the electrostatic coupling effect has very negligible impact (no impact at all) on the threshold voltage and the NoC performance. For comparative performance evaluation, the latency and energy values are normalized with respect to that of the electrostatic-unaware M3D design with 150nm ILD thickness.

Figure 11 shows the effect of ILD thickness on the normalized average latency, energy and EDP of the M3D NoC for all benchmarks with electrostatic coupling-aware and -unaware optimization. From Figure 11(a), it is seen that as the ILD thickness decreases, the latency of M3D NoC increases progressively for both the electrostatic coupling-aware and -unaware optimization. Compared to the unaware M3D NoC, the coupling-aware M3D NoC achieves on an average $14.5 \%, 3.7 \%$, and $1.8 \%$ reduction in latency for 20,50 , and $75 \mathrm{~nm}$ ILD thickness, respectively. There is no latency difference for 100 and 150nm configurations. As the ILD thickness increases, the coupling-aware optimization captures the delay of multi-tier routers and reduces performance penalty by lowering the number of multi-tier routers significantly. From Figure 11(b), we can observe that the energy also increases for both the electrostatic coupling-aware and -unaware designs as the ILD thickness decreases. Compared to the unaware M3D NoC, the coupling-aware M3D NoC achieves on average of $61.1 \%, 4.1 \%$, and $2.7 \%$ reduction in energy dissipation for 20,50 , and $75 \mathrm{~nm}$. Also, there is almost no energy difference for 100 and $150 \mathrm{~nm}$ configuration. While the latency and energy degradations are moderate for the ILD thickness of 50 and $75 \mathrm{~nm}$, the effects are much severe for $20 \mathrm{~nm}$ ILD thickness. The reason behind the significant increase in the latency and energy is that the threshold 


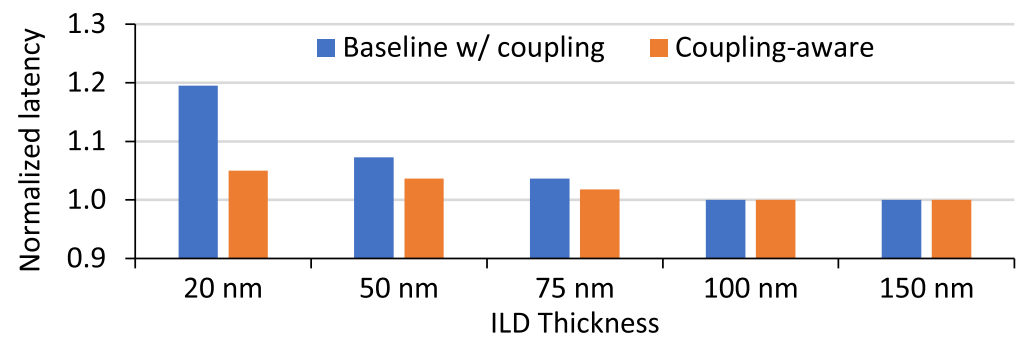

(a) Latency of the M3D NoC with electrostatic coupling-aware and -unaware optimization.

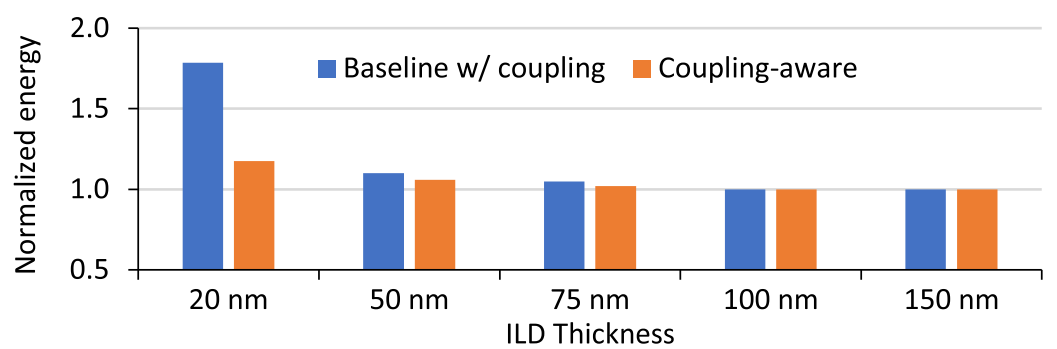

(b) Energy of the M3D NoC with electrostatic coupling-aware and -unaware optimization.

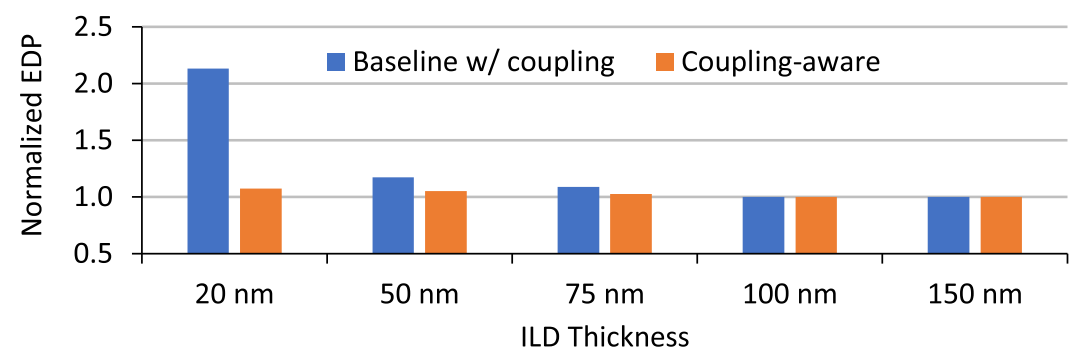

(c) EDP of the M3D NoC with electrostatic coupling-aware and -unaware optimization.

Fig. 11. Effect of ILD thickness on the normalized average (a) latency, (b) energy, and (c) EDP of the M3D NoC with electrostatic coupling-aware and -unaware optimization.

voltage is in inverse proportion to the gate delay (as shown in Equation (2) above). Therefore, there is significant degradation on latency and energy in $20 \mathrm{~nm}$ ILD thickness. In addition, from Figures $11(\mathrm{a})$ and $11(\mathrm{~b})$, the improvement for 50 and $75 \mathrm{~nm}$ thickness is insignificant compared to $20 \mathrm{~nm}$ case This happens due to the fact that the scope of performance improvement for 50 and $75 \mathrm{~nm}$ ILD thickness are considerably less than that for the $20 \mathrm{~nm}$ case.

Figure 11(c) shows the effect of ILD thickness on the normalized average EDP of the M3D NoC with electrostatic coupling-aware and -unaware optimization. As seen from Figures 11(a) and 11(b), as the ILD thickness decreases, the latency and energy consumption increase. Consequently, following the latency and energy trend in Figures 11(a) and 11(b), the EDP also increases progressively as the ILD thickness decreases. Compared to the unaware M3D NoC, the coupling-aware M3D NoC achieves on an average $105.9 \%, 12.5 \%$, and $6.2 \%$ reduction in latency for 20,50 , and $75 \mathrm{~nm}$, respectively. If the ILD thickness is more than $100 \mathrm{~nm}$, then there is no EDP difference between the electrostatic coupling-aware and -unaware optimization. 


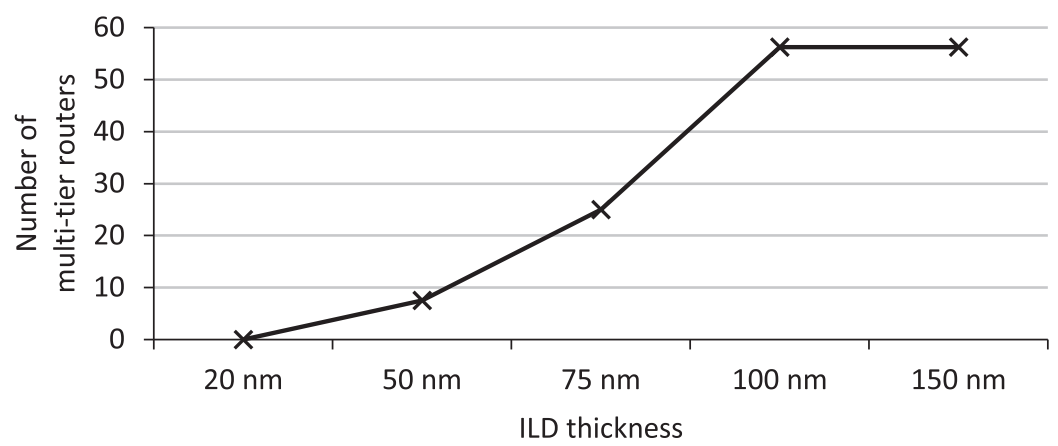

Fig. 12. Average number of multi-tier routers with electrostatic coupling-aware M3D NoC for different ILD thickness for all benchmarks.

The coupling-aware M3D NoC also lowers the number of multi-tier routers and mitigate the delay and coupling effects of multi-tier routers. Figure 12 shows the average number of multi-tier routers with electrostatic coupling aware-M3D NoC for different ILD thickness. In 100 and 150nm cases, there are large number of muti-tier routers in the optimized $\mathrm{NoC}$ to exploit benefits to lower the hop-count and energy dissipation without any penalty. However, as the ILD thickness becomes lower than $100 \mathrm{~nm}$, the number of multi-tier routers decrease due to the trade-off between the performance benefits and electrostatic coupling effect on multi-tier routers. For 50nm ILD thinkness, the average number of multi-tier routers reduces to 8 . When the ILD thickness is lowered below $20 \mathrm{~nm}$, the adverse effect of electrostatic coupling on the multi-tier routers becomes so significant that it offsets any benefits offered by them. Consequently, for 20nm ILD thinkness, the average number (averaged over all the benchmarks considered in this work) of multi-tier routers becomes zero.

\section{CONCLUSIONS}

With the aggressive scaling of inter-layer dielectric thickness, the electrostatic coupling has emerged as a crucial aspect of designing circuits and systems using M3D integration. In this work, we mainly target the trade-off between the performance and power benefits of monolithic 3D integration and electrostatic coupling effect in M3D NoC. For SPLASH-2 and PARSEC benchmarks, electrostatic coupling degrades the EDP on an average by around $18 \%$. Subsequently, to improve the performance of the $\mathrm{M} 3 \mathrm{D}$ NoC, we studied an electrostatic coupling-aware design and optimization approach to efficiently determine the number of multi-tier routers in the M3D NoC. We showed that the coupling-aware design approach reduces the number of allowable multi-tier routers. In addition, we demonstrate the performance of coupling-aware optimization by varying the ILD thickness. The proposed approach minimizes the performance penalty of the M3D NoC compared to the electrostatic coupling-unaware design.

\section{REFERENCES}

K. Acharya, K. Chang, B. W. Ku, S. Panth, S. Sinha, B. Cline, G. Yeric, and S. K. Lim. 2016. Monolithic 3D IC design: Power, performance, and area impact at 7nm. In Proceedings of the 17th International Symposium on Quality Electronic Design (ISQED'16). 41-48.

F. Andrieu, R. Berthelon, R. Boumchedda, G. Tricaud, L. Brunet, P. Batude, B. Mathieu, E. Avelar, A. Ayres de Sousa, G. Cibrario, O. Rozeau, J. Lacord, O. Billoint, C. Fenouillet-Béranger, S. Guissi, D. Fried, P. Morin, J. P. Noel, B. Giraud, S. Thuries, F. Arnaud, and M. Vinet. 2017. Design technology co-optimization of 3D-monolithic standard cells and SRAM exploiting dynamic back-bias for ultra-low-voltage operation. In Proceedings of the IEEE International Electron Devices Meeting (IEDM'17). 20.3.1-20.3.4. 
I. Akturk and O. Ozturk. 2013. ILP-Based communication reduction for heterogeneous 3D network-on-chips. In Proceedings of the 21st Euromicro International Conference on Parallel, Distributed, and Network-Based Processing. 514-518.

S. Bandyopadhyay, S. Saha, U. Maulik, and K. Deb. 2008. A simulated annealing-based multiobjective optimization algorithm: AMOSA. Trans. Evol. Comp 12, 3 (2008), 269-283.

P. Batude, M.-A. Jaud, O. Thomas, L. Clavelier, A. Pouydebasque, M. Vinet, S. Deleonibus, and A. Amara. 2008. 3D CMOS integration: Introduction of dynamic coupling and application to compact and robust 4T SRAM. In Proceedings of the IEEE International Conference on Integrated Circuit Design and Technology and Tutorial. 281-284.

P. Batude, T. Ernst, J. Arcamone, G. Arndt, P. Coudrain, and P. Gaillardon. 2012. 3-D sequential integration: A key enabling technology for heterogeneous co-integration of new function with CMOS. IEEE 7. Emerg. Select. Top. Circ. Syst. 2, 4 714-722.

P. Batude, L. Brunet, C. Fenouillet-Beranger, F. Andrieu, J.-P. Colinge, D. Lattard, E. Vianello, S. Thuries, O. Billoint, P. Vivet, C. Santos, B. Mathieu, B. Sklenard, C.-M. V. Lu, J. Micout, F. Deprat, E. Avelar Mercado, F. Ponthenier, N. Rambal, M.-P. Samson, M. Cassé, S. Hentz, J. Arcamone, G. Sicard, L. Hutin, L. Pasini, A. Ayres, O. Rozeau, R. Berthelon, and F. Nemouchi. 2017. 3D Sequential integration: Application-driven technological achievements and guidelines. In Proceedings of the IEEE International Electron Devices Meeting (IEDM'17). 3.1.1-3.1.4.

P. Batude, M. Vinet, C. Xu, B. Previtali, C. Tabone, C. Le Royer, L. Sanchez, L. Baud, L. Brunet, A. Toffoli, F. Allain, D. Lafond, F. Aussenac, O. Thomas, T. Poiroux, and O. Faynot. 2011. Demonstration of low temperature 3D sequential FDSOI integration down to 50nm gate length. In Proceedings of the Symposium on VLSI Technology-Digest of Technical Papers. 158-159.

C. Bienia. 2011. Benchmarking modern multiprocessors. Ph.D. Dissertation, Princeton University, Princeton, NJ.

N. Binkert, S. Sardashti, R. Sen, K. Sewell, M. Shoaib, N. Vaish, M. D. Hill, D. A. Wood, B. Beckmann, G. Black, S. K. Reinhardt, A. Saidi, A. Basu, J. Hestness, D. R. Hower, and T. Krishna. 2011. The gem5 simulator. ACM SIGARCH Comput. Archit. News 39, 1 (2011).

S. Bobba, A. Chakraborty, O. Thomas, P. Batude, and G. d. Micheli. 2013. Cell transformations and physical design techniques for 3D monolithic integrated circuits. F. Emerg. Technol. Comput. Syst. 9, 3 (2013).

S. Das, J. R. Doppa, P. P. Pande, and K. Chakrabarty. 2017. Monolithic 3D-enabled high performance and energy efficient network-on-chip. In Proceedings of the IEEE International Conference on Computer Design (ICCD'17). 233-240.

S. Das, D. Lee, D. H. Kim, and P. P. Pande. 2015. Small-world network enabled energy efficient and robust 3D NOC architectures. In Proceedings of the 25th Edition on Great Lakes Symposium on VLSI (GLSVLSI'15). ACM, New York, $133-138$

W. R. Davis, J. Wilson, S. Mick, J. Xu, H. Hua, C. Mineo, A. M. Sule, M. Steer, and P. D. Franzon. 2005. Demystifying 3D ICs: The pros and cons of going vertical. IEEE Des. Test 22, 6 (2005), 498-510.

T. Ernst, S. Cristoloveanu, G. Ghibaudo, T. Ouisse, S. Horiguchi, Y. Ono, Y. Takahashi, and K. Murase. 2003. Ultimately thin double-gate SOI MOSFETs. IEEE Trans. Electron. Devices 50, 3 (2003) 830-838.

N. Golshani, J. Derakhshandeh, R. Ishihara, C. I. M. Beenakker, M. Robertson, and T. Morrison. 2010. Monolithic 3D integration of SRAM and image sensor using two layers of single grain silicon. In Proceedings of the IEEE International 3D Systems Integration Conference (3DIC'10). 1-4.

C. Grecu, P. P. Pande, A. Ivanov, and R. Saleh. 2004. Structured interconnect architecture: A solution for the non-scalability of bus-based SoCs. In Proceedings of the 14th ACM Great Lakes Symposium on VLSI (GLSVLSI'04). ACM, New York, NY, 192-195.

T. Hiramoto, T. Saito, and T. Nagumo. 2003. Future electron devices and SOI technology-Semi-planar SOI MOSFETs with sufficient body effect. Jpn. F. Appl. Phys. 42, 1975-1978.

K. Jeong, A. B. Kahng, and K. Samadi. 2009. Impact of guardband reduction on design outcomes: A quantitative approach. IEEE Trans. Semicond. Manufact. 22, 4 (2009), 552-565.

S. Kirpatrick, C. D. Gelatt, and M. P. Vecchi. 1983. Optimization by simulated annealing. Science 220, 4598 (1983), 671-680.

R. G. Kim, W. Choi, Z. Chen, P. P. Pande, D. Marculescu, and R. Marculescu. 2016a. Wireless NoC and dynamic VFI codesign: Energy efficiency without performance penalty. IEEE Trans. Very Large Scale Integr. Syst. 24 (2016) 2488-2501.

K. M. Kim, S. Sinha, B. Cline, G. Yeric, and S. K. Lim. 2016b. Four-tier monolithic 3D ICs: Tier partitioning methodology and power benefit study. In Proceedings of the 2016 International Symposium on Low Power Electronics and Design (ISLPED'16). ACM, New York, NY, 70-75.

J. U. Knickerbocker, P. S. Andry, B. Dang, R. R. Horton, M. J. Interrante, C. S. Patel, R. J. Polastre, K. Sakuma, R. Sirdeshmukh, E. J. Sprogis, S. M. Sri-Jayantha, A. M. Stephens, A. W. Topol, C. K. Tsang, B. C. Webb, and S. L. Wright. 2008. Threedimensional silicon integration. IBM f. Res. Dev. 52, 6 (Nov. 2008), 553-569.

M. Khayambashi, P. M. Yaghini, A. Eghbal, and N. Bagherzadeh. 2015. Analytical reliability analysis of 3D NoC under TSV failure. F. Emerg. Technol. Comput. Syst. 11, 443 (2015).

A. Koneru, S. Kannan, and K. Chakrabarty. 2017. Impact of electrostatic coupling and wafer-bonding defects on delay testing of monolithic 3D integrated circuits. J. Emerg. Technol. Comput. Syst. 13, 4 (2017) 54 (2017), 23 pages. 
Y.-J. Lee, D. Limbrick, and S. K. Lim. 2013. Power benefit study for ultra-high density transistor-level monolithic 3D ICs. In Proceedings of the Design Automation Conference (DAC'13). ACM, New York, NY.

Y.-J. Lee, P. Morrow, and S. K. Lim. 2012. Ultra high density logic designs using transistor-level monolithic 3D integration. In Proceedings of the International Conference on Computer-Aided Design (ICCAD'12). 539-546.

M. Lin, A. E. Gamal, Y.-C. Lu, and S. Wong. 2006. Performance benefits of monolithically stacked 3D-FPGA. In Proceedings of the International Symposium on Field programmable gate arrays (FPGA'06). 113-122.

C. Liu, T. Song, J. Cho, J. Kim, J. Kim, and S. K. Lim. 2011. Full-chip TSV-to-TSV coupling analysis and optimization in 3D IC. In Proceedings of the 48th Design Automation Conference (DAC'11). ACM, New York, NY, 783-788.

O. Lysne, T. Skeie, S. A. Reinemo, and I. Theiss. 2006. Layered routing in irregular networks. In IEEE Trans. Parallel Distrib. Syst. 17 (2006), 51-65.

E. J. Marinissen and Y. Zorian. 2009. Testing 3D chips containing through-silicon vias. In Proceedings of the International Test Conference. 1-11.

D. K. Nayak, S. Banna, S. K. Samal, and S. K. Lim. 2015. Power, performance, and cost comparisons of monolithic 3D ICs and TSV-based 3D ICs. In Proceedings of the IEEE SOI-3D-Subthreshold Microelectronics Technology Unified Conference. $1-2$

S. Panth, K. Samadi, Y. Du, and S. K. Lim. 2014. Power-performance study of block-level monolithic 3D-ICs considering inter-tier performance variations. In Proceedings of the 51st ACM/EDAC/IEEE Design Automation Conference (DAC'14). New York, NY.

L.-S. Peh and W. J. Dally. 2001. A delay model for router microarchitectures. IEEE Micro 21, 1 (2001) 26-34.

J. M. Rabaey, A. Chandrakasan, and B. Nikoli'c. 2003. Digital Integrated Circuits-A Design Perspective. Prentice-Hall, Englewood Cliffs, NJ.

M. Sabry, M. Gao, G. Hills, C. Lee, G. Pitner, M. M. Shulaker, T. F. Wu, M. Asheghi, J. Bokor, F. Franchetti, K. E. Goodson, C. Kozyrakis, I. Markov, K. Olukotun, L. Pileggi, E Pop, J. Rabaey, C. Ré, H.-S. P. Wong, and S. Mitra. 2015. Energyefficient abundant-data computing: The N3XT 1,000x. IEEE Comput. 48.12: 24-33.

S. K. Samal, D. Nayak, M. Ichihashi, S. Banna, and S. K. Lim. 2016. Monolithic 3D IC vs. TSV-based 3D IC in 14nm FinFET technology. In Proceedings of the IEEE SOI-3D-Subthreshold Microelectronics Technology Unified Conference (S3S'16). 1-2.

C. Seiculescu, S. Murali, L. Benini, and G. De Micheli. 2009. SunFloor 3D: A tool for networks on chip topology synthesis for 3D systems on chips. In Proceedings of the Conference on Design, Automation and Test in Europe (DATE'09). 9-14.

G. Van der Plas, P. Limaye, I. Loi, A. Mercha, Herman Oprins, C. Torregiani, S. Thijs, D. Linten, M. Stucchi, G. Katti, D. Velenis, V. Cherman, B. Vandevelde, V. Simons, I. De Wolf, R. Labie, D. Perry, S. Bronckers, N. Minas, M. Cupac, W. Ruythooren, J. V. Olmen, Al. Phommahaxay, M. Broeck, A. Opdebeeck, M. Rakowski, B. D. Wachter, M. Dehan, M. Nelis, R. Agarwal, A. Pullini, F. Angiolini, L. Benini, W. Dehaene, Y. Travaly, E. Beyne, and P. Marchal. 2011. Design issues and considerations for low-cost 3-D TSV IC technology. IEEE 7. Solid-State Circ. 46 293-307.

S. C. Woo, M. Ohara, E. Torrie, J. P. Singh, and A. Gupta. 1995. The SPLASH-2 programs: Characterization and methodological considerations. In Proceedings of the 22nd Annual International Symposium on Computer Architecture (ISCA'95). ACM, New York, NY. 24-36.

Y. S. Yu, S. Panth, and S. K. Lim. 2016. Electrical coupling of monolithic 3-D inverters. IEEE Trans. Electron. Devices 63, 8 (2016), 3346-3349.

Received November 2018; revised June 2019; accepted July 2019 